\title{
Intelligent controlling microbubble radial oscillations by using Slave-Master Feedback control
}

\author{
S. Behnia ${ }^{\mathrm{a}, *}$, M. Yahyavi $^{\mathrm{b}}$, F. Mobadersani $^{\mathrm{c}}$ \\ a Department of Physics, Urmia University of Technology, Urmia, Iran \\ ${ }^{\mathrm{b}}$ Department of Physics, Bilkent University, TR-06800 Bilkent, Ankara, Turkey \\ 'Department of Mechanics, Urmia University of Technology, Urmia, Iran
}

\section{A R T I C L E I N F O}

\section{Keywords:}

Microbubble contrast agents (UCA)

Keller-Herring equation

Slave-Master Feedback (SMF)

Chaos suppression

High intensity focused ultrasound (HIFU)

\begin{abstract}
A B S T R A C T
Dynamics of acoustically driven microbubbles in ultrasonic fields are known to be complex and uncontrollable phenomena indicative of a highly active nonlinear as well as chaotic behavior. In this paper, a method based on Slave-Master Feedback (SMF) to suppress unstable radial oscillations of contrast agents is presented. In the proposed control process, the encapsulated microbubbles as the slave system is coupled with a dynamical system as the master, so that the output of the coupled system is able to produce a stable oscillation. A great virtue of this control technique is its flexibility. In comparison with existing techniques, the present dynamical chaos control method does not need to know more than one variable. The numerical results show its strong impact on reducing the chaotic oscillations to regular ones.
\end{abstract}

(c) 2014 Elsevier Inc. All rights reserved.

\section{Introduction}

In medical sector ultrasound is used in different domains and the application of ultrasound contrast agents (UCAs) is one of the promotion sectors. UCAs are constituted from microbubbles filled with gas by a stabilizing layer such as albumin, polymer or lipids, only a few microns in diameter (1- to $10-\mu$ m diameter) usually administered to a patient by intravenous injection [1-3]. One of main applications of UCAs is to develop the imaging or the blood flow [4]. There were some other cases in which the employment of microbubble is considered for therapeutic application [5-8]. Furthermore, lots of studies in different areas investigate the microbubbles behavior in the acoustic field, but experts are still in the research stage [9-13]. A modern therapeutic technique investigated the active controlling of the effect of the microbubbles. Therapeutic application demanded a High Intensity Focused Ultrasound (HIFU). It is used in various therapeutic applications, such as in cancer treatment [14-16] and tissue coagulation by the microbubbles heat energy [17-19]. Several investigations demonstrated that the echoes after microbubble-assisted HIFU are different from those after HIFU. More recently, Chunga et al. [20] concerned about the increasing effects of the microbubble contrast agent SonoVue on HIFU demolition. The authors of Ref. [21], in both in vitro and in vivo surveys stated that a microbubble contrast agent has the ability to intensify the heating result in treating HIFU. The dynamics of bubble formation and collapse have been studied by using several models containing the RayleighPlesset (RP), Keller and Herring models (K-H) [22]. Prosperetti and Lezzi [23] proposed the Keller-Herring model that can be changed to other models in turn by choosing a judicious parameter. One of the primary efforts was explained by de Jong et al.

\footnotetext{
* Corresponding author.

E-mail address: s.behnia@sci.uut.ac.ir (S. Behnia).
} 
[24], who took the RP equation form and included terms which would account for the elastic shell. Church [25] reformed the original RP equation to consider a bubble encased through a shell. Morgan et al. [26] developed the modified Herring model for UCAs, suggested by Vokurka [27], based on shell modeling of Church and earlier work by Glazman [28]. Macdonald et al. [29] followed the attempts of Prosperetti and Lezzi and Morgan et al. in incorporating the effects of the shell into the K-H equation.

Consequently, it is important to develop techniques for studying the microbubble radial stability in the distinctive situation [30-32]. For this reason, it is substantial to provide appropriate information about the microbubbles dynamics, for finding an acceptable control process in various applications in industry. Having a proper knowledge about microbubbles movement, is the main key of controlling chaotic behavior of the microbubble and using them. In the above-mentioned applications, we should consider that for obtaining the best performance from a microbubble it is necessary to reduce the chaotic oscillations, because when the motion of microbubble gets chaotic, its behavior becomes unpredictable. Therefore, reducing chaotic oscillations could be the first step in controlling the microbubble dynamics by providing more accurate predictions.

The main argument of the paper is the stability analysis of the encapsulated microbubble and the preference of most suitable control parameter for establishing an adequate control strategy based on the Slave-Master Feedback (SMF) method [33]. Actually, we have investigated stability of the microbubble pulsations by controlling the applied acoustic amplitudes. Dynamical frequency of the acoustic has been selected as implementation tools of SMF control method. This frequency, which is used to stabilize the microbubble pulsations (slave system), is generated based on the continuous form of the hierarchy of one-parameter chaotic maps (master system). Then the idea of the coupling is to use the output of the master system to control chaotic pulsations of the slave system. An important advantage of SMF method is that it is enough to have information only for one variable. We have studied the dynamical behavior of the UCA microbubble as a function of acoustic pressure and initial microbubble radius, before and after applying the SMF control method. It has been shown that in some ranges of the acoustic pressure and initial microbubble radius, the microbubble pulsations may be unstable. We demonstrated that the dynamics of the microbubble pulsations, in these ranges, could be controlled to a periodic state by applying the SMF control technique. All the numerical results demonstrate that, the proposed scheme for controlling chaos in the radial response of UCA microbubbles is very effective. The proposed strategy is evaluated through the bifurcation diagrams and the Lyapunov exponent spectra.

\section{Keller-Herring (K-H) models for UCA}

In this study, the radial dynamics of UCA microbubble are modeled by using the general K-H equation [22], derived by Prosperetti and Lezzi [23]. This justified equation also explains the effect of variation of the shell on the UCA behavior. This class of models contains the elastic shell which makes the microbubble to represent nonlinear acoustic properties [35]. The $\mathrm{K}-\mathrm{H}$ equation for an UCA with a thin elastic shell is given by the following equation:

$$
\left[1-(1+\beta) \frac{\dot{R}}{c}\right] R \ddot{R}+\frac{3}{2}\left[1-(3 \beta+1) \frac{\dot{R}}{3 c}\right] \dot{R}^{2}=\frac{1}{\rho}\left[1+(1-\beta) \frac{\dot{R}}{c}+\frac{R}{c} \frac{d}{d t}\right] \times\left[P(R, \dot{R})-P_{\infty}(t)\right],
$$

with an explicit expression for $P(R, \dot{R})$ which developed by Morgan et al. [26], allowing for the encapsulating shell, is defined as:

$$
\left\{\begin{array}{l}
P(R, \dot{R})=\left(P_{0}+\frac{2(\sigma+\gamma)}{R_{0}}\right)\left(\frac{R_{0}}{R}\right)^{3 \Gamma}-\frac{4 \mu \dot{R}}{R}-\frac{2 \sigma}{R_{0}}-\frac{2 \chi}{R}\left(\frac{R_{0}}{R}\right)^{2}-12 \mu_{s h} \varepsilon \frac{\dot{R}}{R(R-\varepsilon)}, \\
P_{\infty}(t)=P_{0}+P_{a c}(t) \quad \text { where } \quad P_{a c}(t)=P_{a} \sin (2 \pi f t) .
\end{array}\right.
$$

In this equation $R=R(t)$ is the UCA's radius, $R_{0}$ is the initial radius, $\mu_{s h}$ is the viscosity of the shell, $\varepsilon$ is the shell thickness, $\mu$ is the viscosity of the liquid, $\Gamma$ is the non-dimensional polytropic gas constant of the gas inside the UCA, $\chi$ is the shell elasticity, $\sigma$ is the surface tension and $P(R, \dot{R})$ demonstrates the pressure on the liquid aspect of the interface for an isolated UCA microbubble [35,36]. The expression $P_{\infty}(t)$ shows the pressure in the liquid far from the microbubble, with $P_{0}$ being the ambient static pressure and $P_{a c}(t)$ the acoustic forcing term. The model was solved for isolated bubble using the values of the physical constants which represented in Table 1 for Albunex [35,24,25].

Setting the arbitrary parameter $\beta=0$ and $\beta=1$ respectively give Keller and Herring-type equation, but Prosperetti and Lezzi [22] studied that these two equations exhibit similar characteristics. Also, It is worth noting that with letting $c \rightarrow \infty$ the liquid can be considered as incompressible and then Eq. (1) reduces to the form of a Rayleigh, Plesset, Noltingk, Neppiras and Poritsky (RPNNP) equation for bubble dynamics in an incompressible fluid [22]. Our main purpose of employing K-H model is its ability in generating results in best way with those achieved by solving the full partial differential equations of fluid dynamics, also it is appropriate for a wide range of amplitudes of oscillations [23]. Note that in this article we concentrate on the Herring model $(\beta=1)$ in particular widely used in the literature [37-39] to simulate the radial response of UCA microbubble in an acoustic pressure field. 
Table 1

Constant parameters used in the general Keller-Herring equation for an ultrasound contrast agent microbubble (for a bubble/water system at $20^{\circ} \mathrm{C}$ ).

\begin{tabular}{llll}
\hline Symbol & Description & Units & Value \\
\hline$\mu$ & Viscosity & $\mathrm{Ns} / \mathrm{m}^{2}$ & 0.001 \\
$\sigma$ & Surface tension & $\mathrm{N} / \mathrm{m}$ & 0.072 \\
$c$ & Sound velocity & $\mathrm{m} / \mathrm{s}$ & 1481 \\
$p_{0}$ & Static ambient pressure & $\mathrm{N} / \mathrm{m}^{2}$ & $1.01 \times 10^{5}$ \\
$\rho$ & Liquid density & $\mathrm{kg} / \mathrm{m}^{3}$ & 998 \\
$\chi$ & Shell elasticity & $\mathrm{N} / \mathrm{m}$ & 8 \\
$\varepsilon$ & Shell thickness & $\mathrm{m}$ & $15 \times 10^{-9}$ \\
$\mu_{\text {sh }}$ & Shell viscosity & Pa s & 1.77 \\
$\Gamma$ & Polytropic exponent for the encapsulated gas & & 1.33 \\
\hline
\end{tabular}

\section{Slave-Master Feedback control}

In recent years, the explanation of the modern methods of nonlinear dynamical systems has been developed in recognizing the nonlinear behavior of microbubbles and encapsulated microbubbles [35,40-43]. Lately, researchers give more attention to this investigation, so that the study of their behavior has recently been the center of attention. It is believed that this phenomenon exhibits highly complex and chaotic dynamics both numerically [35,43,44] and experimentally [45-47]. Recognizing the values of physical parameters that determine chaotic oscillations importance which are useful in defining the insonating field to empower enable controlled bubble in the clinical application. Most recently, the following methods of control have been proven to be successful in the controlling chaotic oscillations of microbubble:

- Dual forcing frequency (through applying a periodic perturbation [48]).

- Varying bubble cluster size (the effects of coupling and bubble size [40]).

The first method is usually presented a technique based on using periodic perturbation to suppress chaotic oscillations of a spherical cavitation bubble. The second method theoretically has focused on the suppression of chaos in the dynamics behavior of a small cluster of microbubbles.

Recently, the problem of controlling chaos has been attracted [49-53]. It is appropriate to decompose the practical attempts on chaos into scientific and technical (engineering) applications. Most of the studies confirmed that the following methods of control are profitable in the experimental control of chaos.

- Identification of the stable and unstable directions in the Poincaré section.

- Self-controlling feedback procedure.

- Presentation of small modulation of a control parameter.

- Information of an appointed goal dynamics.

The first two methods are usually named feedback techniques and the third and last are named non-feedback methods [49,54]. Small modulation techniques (the third method), theoretically has focused on the suppression of chaos in the dynamics of various models. However, this method has not been proved in general [55], it includes chaotic behavior generated by error signals due to the dissimilarity between the output signal and its value at an earlier time.

Before evaluating the potential chaos control methods for a cavitation microbubble system, we should consider the parameters of the $\mathrm{K}-\mathrm{H}$ models which available to be freely exposed to the control technique. According to the result, we determined that in order to have regular desirable behavior, one of the control parameters of the $\mathrm{K}-\mathrm{H}$ models should be modified. However, in a real world application dealing with cavitation phenomenon, we are restricted to apply changes only to the external forcing term $\left(P_{a c}(t)\right)$. The acoustic pressure amplitude and the frequency of the acoustic are determined by the type of the application. However, despite the extensive employment of the frequency acoustic origin in applications involving cavitation microbubble phenomenon, there are no other researches about estimating the proficiency of control methods by SMF method in a cavitation UCAs microbubble system. With reference to this event attendant improvements with employing frequency of the acoustic, simplicity of engaging and dealing with such methods and more vitally the proven capability of SMF method exploited in practice. In this paper, we preferred to base our control process on the stability analysis of the UCAs microbubble system by electing of most proper control parameter.

For stability analysis, it is convenient to convert the second-order differential equation (1) into an autonomous system of first-order differential equations (see Appendix A). By considering the controlled process obey the following state equations:

$$
J \frac{d V}{d t}=F(V, \alpha)
$$

where $V(x, y, \theta)$ an autonomous vector field and $\alpha\left(R_{0}, P_{0}, P_{a}, f, \mu, \mu_{s h}, \beta, \rho, c, \Gamma, \epsilon, \chi, \sigma\right)$ is an element of the parameter space. This system generates a flow $\Phi=\left\{\Phi^{T}\right\}$ on the phase space $M=R^{2} * S$ and there exists a global map: 


$$
P: \sum_{c} \longrightarrow \sum_{c}, \quad V_{P}(x, y, \theta) \longrightarrow P\left(V_{P}\right)=\left.\left\{\Phi^{T}\right\}\right|_{c}\left(x, y, \theta_{0}\right),
$$

with $\mathrm{T}=\frac{1}{f}, \theta_{0}$ is a constant determining the Poincaré cross-section and $(x, y)$ the coordinates of the attractors in the Poincaré cross-section $\sum_{c}$, which is defined as:

$$
\sum_{c}=\left\{(x, y, \theta) \in R^{2} * S^{1} \mid \theta=\theta_{0}\right\} .
$$

For driven oscillators similar the microbubble model, a natural way to define $\sum$ is to cut the torus like state space $M$ transversally to the cyclic $\theta$ direction at a fixed value $\theta_{0}$ of $\theta[56]$.

The method presented by the authors however, consider the parameter $f$ (frequency of the acoustic) as a time variant variable that its variation can be described by chaotic map [34]:

$$
\dot{f}=\frac{4 f-(1-f)^{2}}{\xi_{t}(1-f)^{2}} .
$$

In SMF control technique, UCAs microbubble dynamic system is called the slave system and another chaotic system [34] (see Eq. (6)) is called the master system, then the idea of the coupling is to use the output of the master system to control the slave system, so that the output of the coupled system is able to generate a stable oscillation. By considering a UCAs microbubble and the control system (Eq. (6)) as a two dimensional dynamical system (Slave-Master system), the simple model for controlling the stability of a UCAs microbubble is introduced as follows:

$$
\frac{d \widetilde{V}}{d t}= \begin{cases}\frac{d V}{d t}=F(V, \beta) & \text { Slave system, } \\ \dot{f}=\frac{4 f-(1-f)^{2}}{\xi_{t}(1-f)^{2}} & \text { Master system, }\end{cases}
$$

where $\xi_{t}$ is an arbitrary number between $(0,1), \widetilde{V}(x, y, f, \theta)$ an autonomous vector field and $\beta\left(R_{0}, P_{0}, P_{a}, \mu, \mu_{s h}, \beta, \rho, c, \Gamma, \epsilon, \chi, \sigma\right)$ is an element of the parameter space. By considering Eq. (A.1) and the control system equation (6) as a four dimensional dynamical system the simple and suitable model for controlling the stability of the Keller-Herring bubble model, given in Eq. (A.2). In the SMF control scheme, the system equation (1) was driven by following acoustic pressure:

$$
P_{d a c}(t)=\left\{\begin{array}{l}
P_{a c}(t)=P_{a} \sin (2 \pi f t), \\
\dot{f}=\frac{4 f-(1-f)^{2}}{\xi_{t}(1-f)^{2}},
\end{array}\right.
$$

where $P_{d a c}(t)$ represents the dynamical acoustic forcing term. As this method is independent of geometrical considerations, so it can easily be applied in high dimensional dynamical systems. So, it makes possible for us to study the interaction of the microbubbles by a controlled technique in which we can realize the microbubble cluster better. The concept of feedback control has been re-defined by taking into account the control parameter as a variable in the time that is changeable by another chaotic map [34], for which a new and effective control scheme has been presented.

\section{Analysis tools}

There are several mathematical tools available for quantifying microbubble stability ranging, the reasons to use maximum Lyapunov exponents and bifurcation structure in the absence of direct mathematical methods are:

- The maximum Lyapunov exponents, approximated computationally for a wide range of injection values, clearly indicates the chaotic behavior of microbubble interaction dynamics.

- The computationally based bifurcation analysis illustrate that the microbubble interaction dynamics transits among different regions such as fixed point, chaotic attractors and intermittent behavior.

\subsection{Computation of Lyapunov exponents}

One of the significant ways for studying the behavior of microbubble dynamics is to calculate the Lyapunov exponent spectrum which is a measure of the sensitivity of the system to initial conditions and the exponential rates of divergence or convergence of nearby trajectories in state space. The Lyapunov exponents, can be considered as "dynamic" measures of attractors complexity and is called "time average" [57]. They are helpful to characterize chaos and bifurcation that are common as nonlinear effects in microbubble dynamics. The Lyapunov exponents are defined as follows:

Consider two nearest neighboring points (usually the nearest) in phase space at time 0 and $t$, with distances of the points in the $i$ - th direction $\left\|\delta x_{i}(0)\right\|$ and $\left\|\delta x_{i}(t)\right\|$, respectively. The Lyapunov exponent is then defined through the average growth rate $\lambda_{i}$ of the initial distance,

$$
\frac{\left\|\delta x_{i}(t)\right\|}{\left\|\delta x_{i}(0)\right\|}=2^{\lambda_{i} t}(t \rightarrow \infty) \quad \text { or } \quad \lambda_{i}=\lim _{t \rightarrow \infty} \frac{1}{t} \log _{2} \frac{\left\|\delta x_{i}(t)\right\|}{\left\|\delta x_{i}(0)\right\|}
$$


There are three possibilities:

- If $\lambda<0$ the trajectories go close to each other $\rightarrow$ stable radial oscillation.

- If $\lambda=0$ the orbits maintain their relative positions, they are on a stable attractor.

- If $\lambda>0$ implies that the orbit never falls within the basin of attraction of any periodic orbits $\rightarrow$ unstable radial oscillation (chaotic behavior).

In the chaotic region, this demonstrates neighboring points with infinitesimal differences at the initial state suddenly separated from suddenly state separation in the ith direction [58]. On the other hand, even if the initial states are nearby, the final states are very different. Hence this phenomenon is sometimes named sensitive dependence on initial conditions. Commonly, Lyapunov exponents $(\lambda)$ can be extracted by observed signals in following different method:

- Based on the opinion of following the time-evolution of nearby points in the state space.

- Based on the estimation of local Jacobi matrices.

The first method is usually called Wolf algorithm [59] and it provides an estimation of the largest Lyapunov exponent only. The second method is capable of estimating all the Lyapunov exponents. Using one of these methods, the Lyapunov exponent is calculated rather than a given control parameter. So, there is a little increase in value of the control parameter and the Lyapunov exponent is calculated for the new control parameter. By continuing this method the Lyapunov exponent spectrum of the microbubble dynamics system is plotted versus the control parameter.

\subsection{Bifurcation diagrams}

Period doubling, quasi-periodicity and intermittency are well known routes of transition from periodic to chaotic behavior with their origins in local bifurcations. A qualitative change in the dynamical behavior of a system, such as dynamics of microbubbles in ultrasonic fields, when a parameter of the system is varied, is called a bifurcation. As it is known, a appropriate method of studding bifurcation is by bifurcation diagram, in that, it provides a helpful insight into the transition between different types of behavior that can occur as one parameter of the system alters.

In this paper, the dynamical behavior of the microbubble radial oscillations is studied by plotting the bifurcation diagrams of the normalized radius of the microbubble in comparison with different control parameters. The analysis of the bifurcation diagram was carried out in the Poincaré section $(P)$. To choose the appropriate Poincaré section, we use the general technique of setting one of the phase space coordinates to zero. In our analysis the following condition was used:

$$
P \equiv \max _{R}\{(R, \dot{R}): \dot{R}=0\}
$$

which gives the maximal radius from each acoustic period. Also, this condition was used to plot the bifurcation diagram of a cavitation bubble in [60]. In order to generate the bifurcation points, the equation of the microbubble motion was solved numerically for 900 acoustic cycles of the lower frequency and then a Poincare section was constructed. By considering just the last 300 cycles convinces that the initial transient behavior is eliminated. For creating bifurcation diagram:

- On the $x$-axis plotted a bifurcation parameter which is varied.

- On the $y$-axis plotted the asymptotic behavior of a sampled state parameter as discrete point $\left(\frac{R}{R_{0}}\right)$.

After the system reached its steady state, up to 600 orbits of $\frac{R}{R_{0}}$ in the condition of $\theta_{0}=0$ Poincaré section, were plotted in the bifurcation diagram versus microbubble control parameter. This method continued through increasing the control parameter and the new resulting discrete points were plotted in the bifurcation diagram versus the new control parameter. For a full discussion on the bifurcation diagram, and Lyapunov exponent spectrum their utilization in order to study the microbubble dynamics, one can refer to [56,61].

\section{Results and discussions}

\subsection{Presenting the dynamics of $\mathrm{K}-\mathrm{H}$ model for $U C A$}

\subsubsection{Effect of acoustic pressure}

We examine the stability of an microbubble $[24,25]$ in ultrasonic field by considering the driving pressure amplitude and the initial radius of the microbubble. Radial motion of single UCA microbubble dynamics is investigated versus a prominent domain of acoustic pressure from $10 \mathrm{kPa}$ to $2 \mathrm{MPa}$. Fig. 1(a) and (b) shows the bifurcation diagrams of the normalized UCA microbubble radius when acoustic pressure of the UCA is taken as the control parameter with the frequency of 3 MHz for several values of initial radii of the UCA which are 3 and $5 \mu \mathrm{m}$, respectively, which stable and chaotic pulsations can be observed in each. Fig. 1 shows the chaotic oscillations of UCA appeared by increasing the values of applied pressure and the microbubble demonstrates more chaotic oscillations as the pressure is intensifying (Fig. 1(a) and (b)). According to 

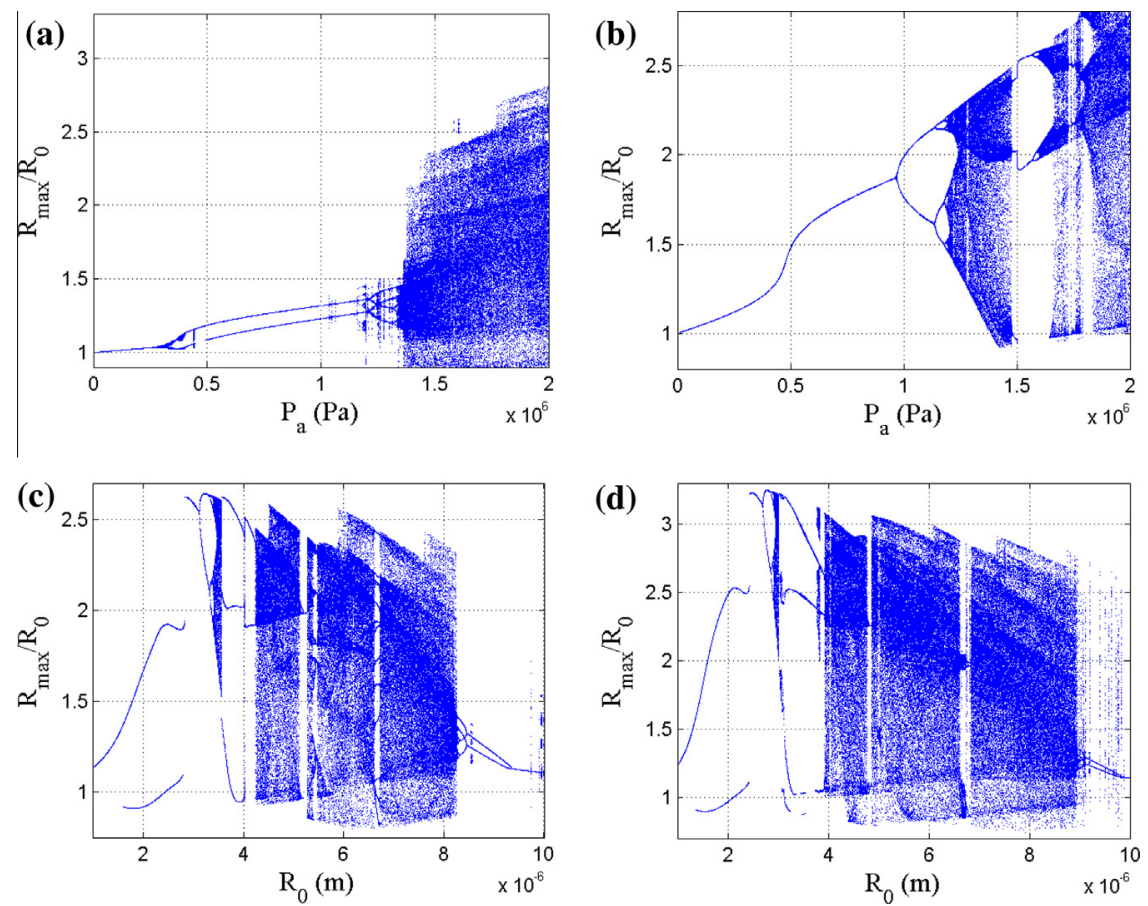

Fig. 1. Bifurcation diagrams of normalized UCA microbubble radius versus pressure ((a) and (b)) and initial radius ((c) and (d)) while, (a) driving frequency is $3 \mathrm{MHz}$ and the initial radius is $3 \mu \mathrm{m}$, (b) driving frequency is $3 \mathrm{MHz}$ and the initial radius is $5 \mu \mathrm{m}$, (c) driving frequency is $2 \mathrm{MHz}$ and the pressure is $1.5 \mathrm{MPa}$, (d) driving frequency is $2 \mathrm{MHz}$ and the pressure is $2 \mathrm{MPa}$.

Fig. 1, the magnitude of pulsations decreases significantly and chaotic region becomes smaller when the initial radius of the microbubble is increasing. The results are in agreement with the previous studies [35,43].

\subsubsection{Effect of initial microbubble radius}

In this section, we examine the variation of initial radius on microbubble dynamics by considering the initial radius as a control parameter. In Fig. 1(c) and (d) we can see the motion of microbubble in ultrasonic fields when driven by the frequency limited $2 \mathrm{MHz}$ and the applied pressure amplitude is 1.5 and $2 \mathrm{MPa}$, respectively. It is observed that the microbubble behavior is stable for low values of pressure. From the results, it can be understood that the motions of microbubble can be chaotic or stable in particular ranges. The results are in agreement with the previous studies, clearly highlighting that microbubbles are dependent on the driving frequency variations [35,41-43]. Most of the results demonstrate the uncontrollable and chaotic motion in UCA microbubble dynamics. In dissimilar situations and values for controlling parameters such as: pressure, frequency and the initial bubble radius, microbubble shows various motions and oscillations by themselves and in addition they change their motion from one type to another. This involves simple period one, transformation by period doubling bifurcation to period two, successive period doublings leading to chaos and high periods, symmetry breaking transition and etc. In order to keep away from forcing dynamical results, it is essential to use control techniques for reducing the chaotic oscillations.

\subsection{Dynamics of a encapsulated spherical microbubble in presence of SMF control process}

In the previous subsection our numerical simulation explicated that the radial oscillations of a contrast agent microbubble can have chaotic behavior. These results reveal that the chaotic oscillations of UCA under the action of an ultrasound field can be used to distinguish stable and unstable regions of microbubble pulsations and the expansion ratio of the UCA. In order to streamline the manifestation of the technique efficiency in suppressing chaos, a few chaotic zones have been chosen as samples to be subjected to the SMF control method. For the correlated zones the dynamical behavior of the microbubble was analyzed before and after the control process. This is done through computing its bifurcation diagram, the corresponding Lyapunov spectrum, Poincaré section of the phase space and time series. The results are depicted in Figs. 2-6.

\subsubsection{Effect of acoustic pressure through applying SMF technique:}

The first sample (pressure-bifurcation diagram of microbubble) is presented in Fig. 2 (blue points). It belongs to a microbubble with initial radius of $5 \mu \mathrm{m}$ exposed to a frequency of the acoustic force of $2 \mathrm{MHz}$ when the control parameter 


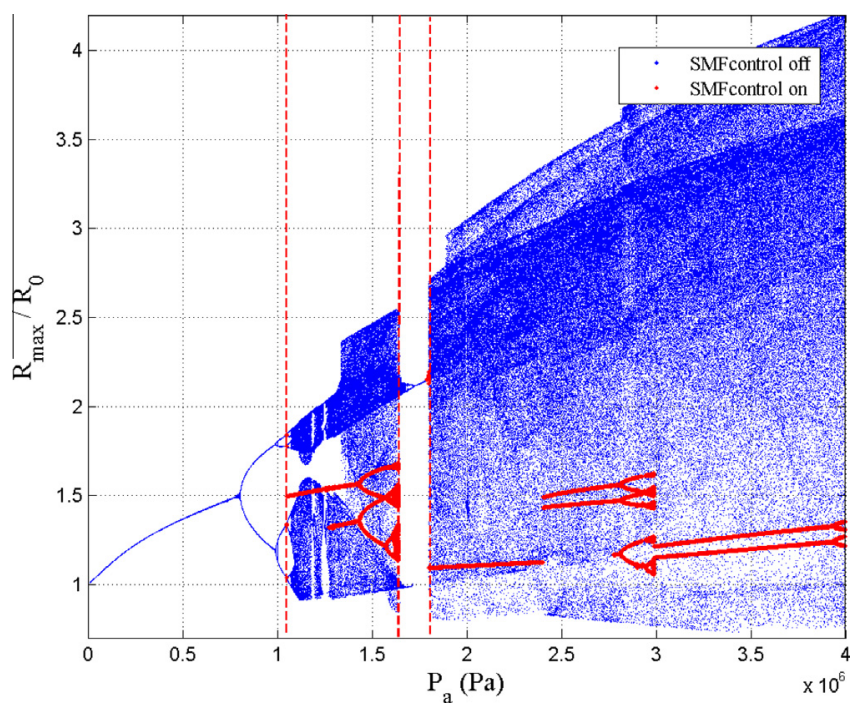

Fig. 2. Bifurcation diagrams of the UCA microbubble radius driven by $2 \mathrm{MHz}$ of frequency with the initial radius of $5 \mu \mathrm{m}$ versus pressure: (blue points) Chaotic behavior before applying the proposed technique, (red points) after applying the SMF control method. (For interpretation of the references to colour in this figure caption, the reader is referred to the web version of this article.)

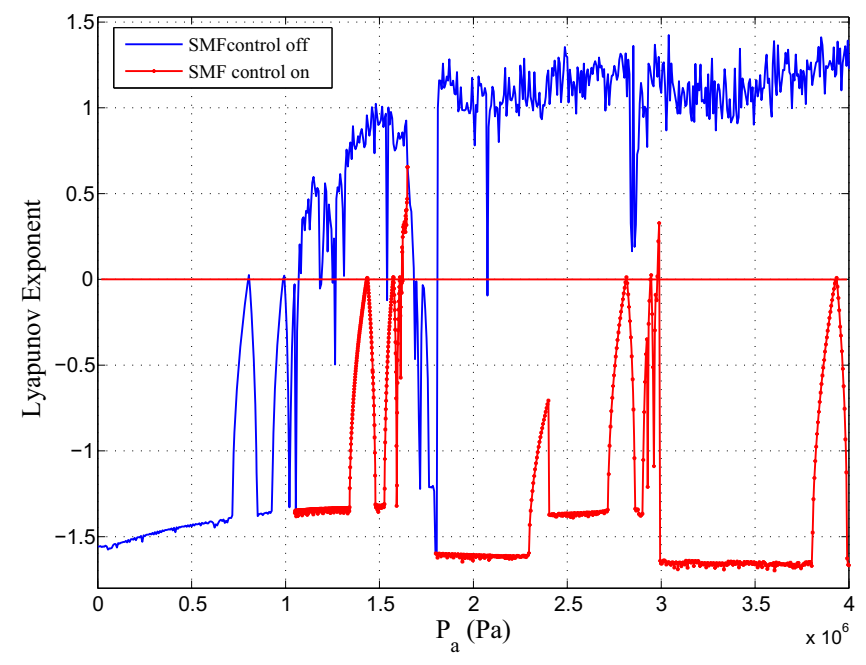

Fig. 3. Lyapunov spectra of the UCA microbubble radius driven by $2 \mathrm{MHz}$ of frequency with the initial radius of $5 \mu \mathrm{m}$ versus pressure: Blue points represent the case before applying the SMF control method while red points represent the system after the SMF control. (For interpretation of the references to colour in this figure caption, the reader is referred to the web version of this article.)

is pressure in the range of $10 \mathrm{kPa}-4 \mathrm{MPa}$ (a condition used typically during HIFU [62,63]). It is easily understood that by increasing pressure amplitudes the microbubble stability is reduced and chaotic oscillations will be obvious which can be proved by previous studies [35,40-43]. In order to study the possibility of reducing chaos in oscillation of UCA microbubble, a dynamical control method is applied. Fig. 2 (red points) gives us some information about controlling dynamics after applying the usage of control method. After the employment of these methods, we observed that the chaotic zone reduces (see Fig. 2). The maximum Lyapunov exponent is also an important indicator for a dynamical system to have a potentially chaotic behavior. Accordingly, the maximum Lyapunov exponents is outlined in Fig. 3. Fig. 3 (blue points) introduces the original system, while Fig. 3 (red points) introduces the controlled system, where Lyapunov exponent is mostly positive showing a chaotic behavior. The negative Lyapunov exponent demonstrates the stable behavior. In order to better understand the SMF control method, the controlling technique has also been assumed by plotting the normalized microbubble oscillations versus time in a certain value of the pressure before and after control in Fig. 4. Fig. 4(a) and (b) indicates the 
(a)

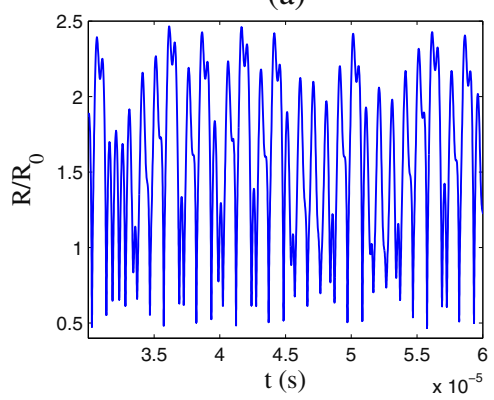

(d)

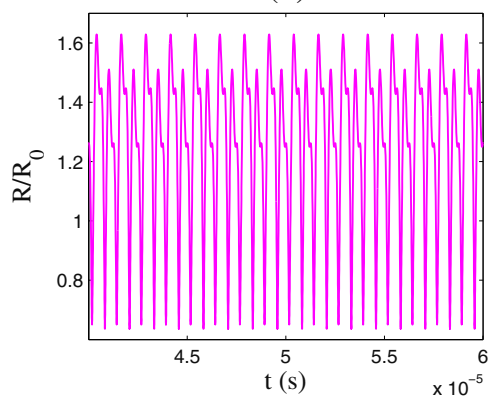

(b)

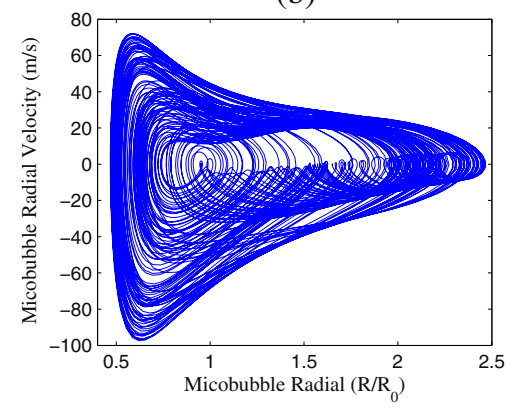

(e)

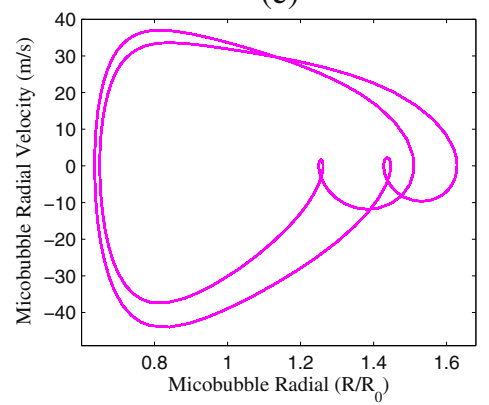

(c)

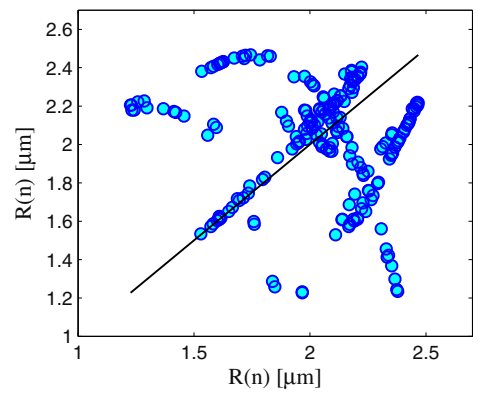

(f)

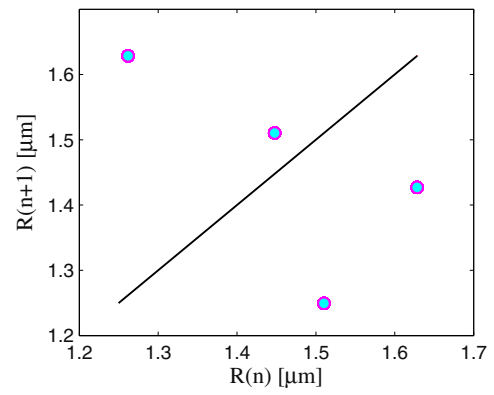

Fig. 4. Time series, trajectory in state space projection and Poincaré section plot of UCA microbubble radius driven by $5 \mu \mathrm{m}$ initial radius and $1.5 \mathrm{MPa}$ of pressure: (a-c) Chaotic oscillations (without applying the proposed technique), (d-f) regular oscillations (after applying the SMF control technique).

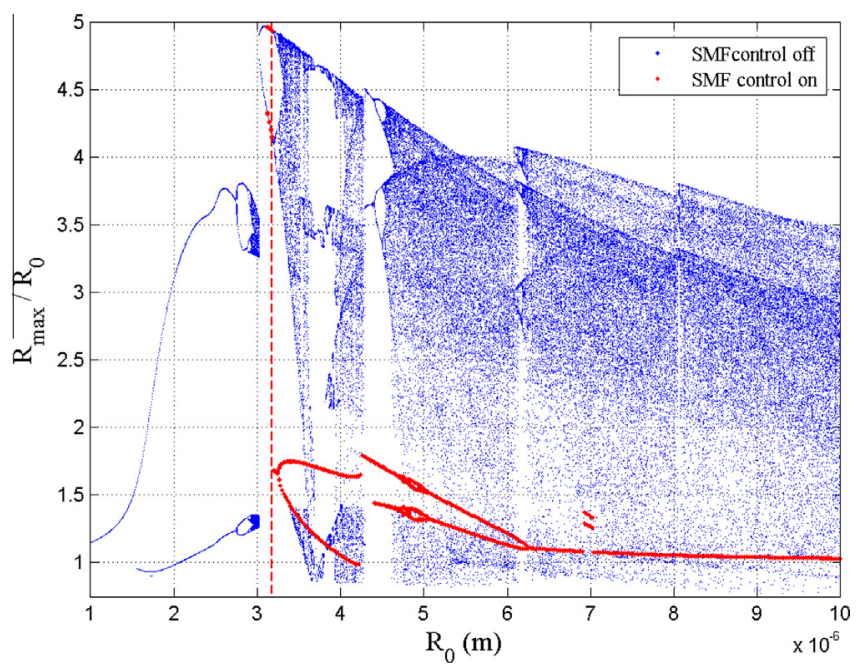

Fig. 5. Bifurcation diagrams of the UCA microbubble radius driven by $1 \mathrm{MHz}$ of frequency with the pressure of $1.5 \mathrm{MPa}$ versus initial radius: (blue points) Chaotic behavior before applying the proposed technique, (red points) after applying the SMF control method. (For interpretation of the references to colour in this figure caption, the reader is referred to the web version of this article.)

chaotic oscillations of microbubble at parameter values ( $P_{a}=1.5 \mathrm{MPa}, R_{0}=5 \mu \mathrm{m}, f=2 \mathrm{MHz}$ ). The orbit projection (Fig. 4(b)) reveals the strange attractor that is created and Fig. 4(c) presents the Poincare cross-section ${ }^{1}$ which does not yet show a complete fractal shape. Therefore, in under control process the oscillations settles onto four stable limit cycles in the state space trajectory (Fig. 4(e)). It is clearly seen in the Poincaré plot for this state (Fig. 4(f)), where four point are created, the doubling

${ }^{1}$ The choice of Poincaré section is arbitrary; the only necessary condition is that the trajectory should cross the plane of the section once every acoustic cycle. 


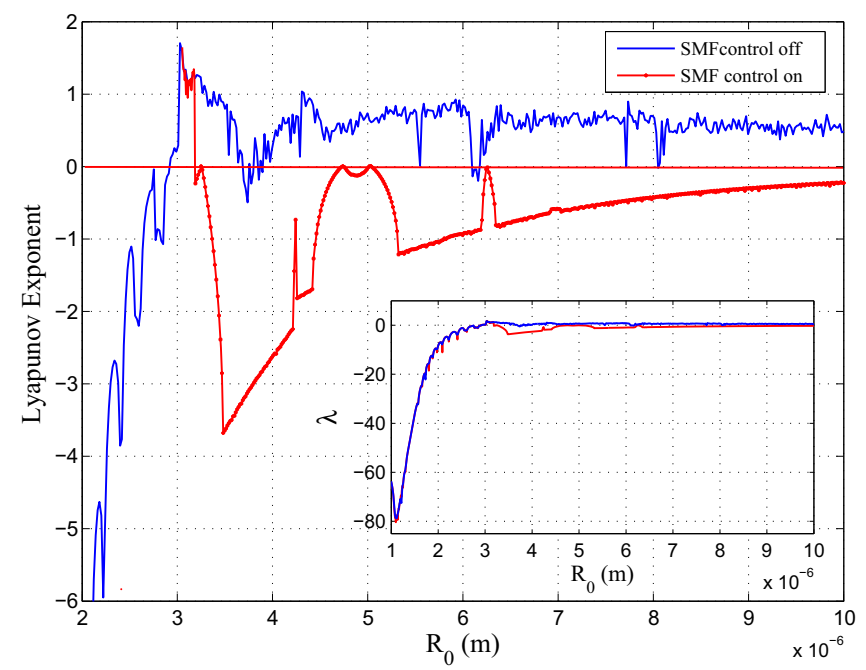

Fig. 6. Lyapunov spectra of the UCA microbubble radius driven by $1 \mathrm{MHz}$ of frequency with the pressure of $1.5 \mathrm{MPa}$ versus initial radius: Blue points represent the case before applying the SMF control method while red points represent the system after the SMF control. (For interpretation of the references to colour in this figure caption, the reader is referred to the web version of this article.)

of the period of oscillation in signified (it takes the solution four driving periods to come back to the same point in space). This is an easy sample of a period-doubling bifurcation of cycles. Comparison of the cases for UCA microbubble with and without SMF control action in Figs. 2-4 indicated that the presence of the SMF controller suppresses oscillations and decreases the nonlinearity of the responses of UCA microbubble.

\subsubsection{Effect of initial radius through applying SMF technique}

By considering the initial radius as a control parameter (size of the microbubbles in treatment of uterine fibroids with HIFU ranges from 1-10 $\mu \mathrm{m}$ [64]) the variation of initial radius on UCA microbubble dynamics has been studied. Fig. 5 (blue points) shows the second chaotic sample zone (radius-bifurcation diagram of UCA microbubble) before applying the dynamical control technique. It is related to a microbubble subjected to a driving frequency source of $1 \mathrm{MHz}$ and pressure amplitude 1.5 MPa, versus its initial radius as the control parameter. As we observe when the initial radii $\left(R_{0}\right)$ is used as the control parameter, a period doubling sequence is followed by a transition to chaos. Fig. 5 (red points) demonstrates the controlled dynamics. Also, the control method is tested through the maximum Lyapunov exponent diagrams (see Fig. 6). This figure illustrates a significant abatement of the maximum Lyapunov exponent from positive values to negative ones indicating that stable behaviors were achieved after the proposed method was engaged. Fig. 6 (blue points) corresponds the original microbubble dynamics system, and Fig. 6 (red points) corresponds the controlled system.

The obtained results indicate that stable dynamics can be achieved after the SMF control technique (see Tables 2 and 3 ). Reducing chaos using SMF control can be practically advantageous, in particular, in applications involving cavitation microbubbles for medical purposes. Introduction of microbubbles has raised HIFU liver tumor destruction through destroying greater volumes after a short exposure [65]. Since, coated microbubbles are filled with gas, they are so compressible. When these microbubbles exposed to an ultrasound field, because of the high pressure they contract more. Particularly, in high

Table 2

Domain of values of parameters that lead to periodic oscillations in the general $\mathrm{K}-\mathrm{H}$ equation before and after applying the SMF method (for a bubble/water system at $20^{\circ} \mathrm{C}$ ). All other physical parameters were kept constant at values given in Table 1.

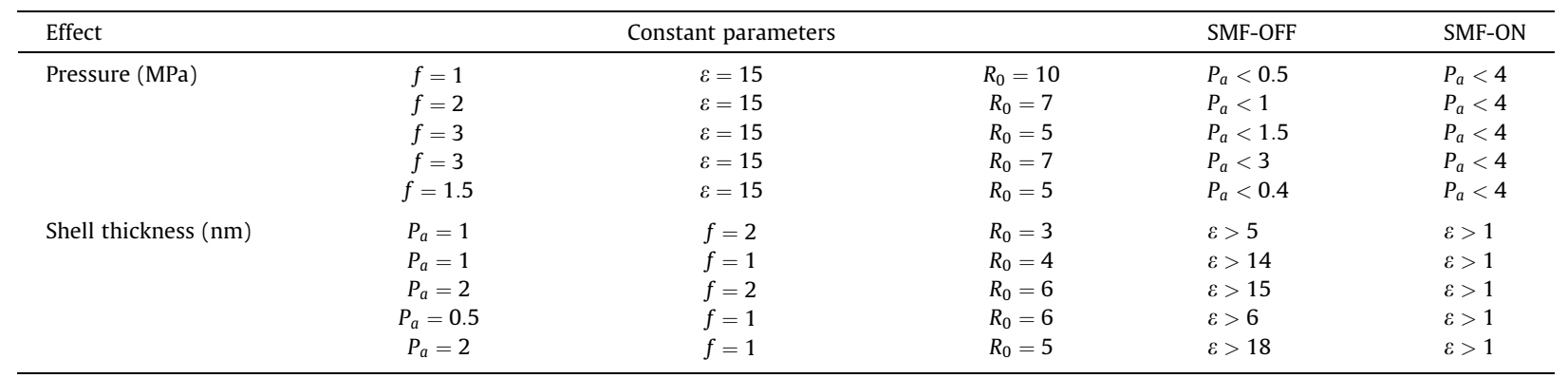


Table 3

Results of the SMF control tests for the domain of values of parameters that lead to chaotic oscillations in the general K-H equation (for a bubble/water system at $20^{\circ} \mathrm{C}$ ). All other physical parameters were kept constant at values given in Table 1 .

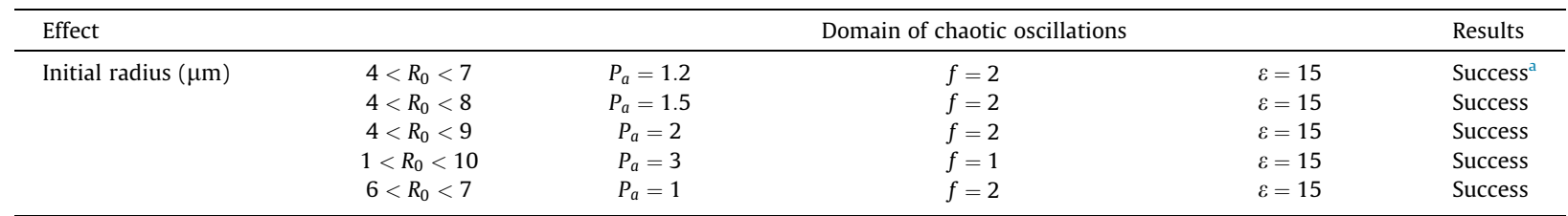

\footnotetext{
a After applying the proposed technique for the physical parameters were kept constant at values given in Tables 1 and 3 , no chaotic radial oscillations
} occurred within $1<R_{0}(\mu \mathrm{m})<10$.

pressure amplitudes, the behavior of microbubbles in the state of the ones necessary for HIFU, is chaotic. Obviously, all of the techniques that can help to decrease the chaotic oscillations are very important, because in this way predictability and safety of treatment increase [9-11,66-68]. In fact, because of this negative impression of chaotic behaviors in treatment, reducing chaotic oscillations of microbubble dynamics can be a primary step in increasing the safety and predictability of the treatment. For examining the capability of SMF method to control chaotic oscillations of microbubbles during HIFU, we have considered the case of the injected microbubbles in the above numerical.

\section{Conclusion and outlook}

This paper the dynamics of acoustically driven shelled microbubble is explained by using the techniques of chaos theory[33,35,43,40-42]. All of the outcomes indicate its nonlinear and chaotic behaviors with respect to the variations in the control parameters of the general Keller-Herring equation [22,23]. So according to the light of the discussion, it can be stated that acoustic forcing term demonstrates its influence on the microbubble dynamics $[35,44,56]$. As a result, acoustic pressure amplitude is one of most important factors in the stability of the microbubble. Practically, frequency of the acoustic is the other important factor in the microbubble dynamics $[33,35,62,63]$. It is also an important factor, which can influence UCA microbubble dynamics. In order to decrease chaotic oscillations of the UCA microbubble, by considering frequency of the acoustic as a practical control technique, the novel and practical control technique based on the frequency is presented. Based on the obtained results it can be concluded that, the application of the presented method in the control of the chaotic domain has an important advantage in the control of the radial response of UCA microbubbles to an acoustic pressure field. This particular control scheme is so prominent since it is adaptable to applications involving cavitation event such as HIFU treatment of brain tumors [7,10,65,69-72]. Expansion of cavitation microbubbles in the treatment site can cause power reduction essential for tumor ablations by immediacy mechanical impress or amplifying the temperature [70].

Consequently, we can select a corresponding control process to match our physical conditions. By focusing on the mechanisms governing the transition from the chaotic oscillations to the stable region, this study opens a new horizon in studying chaotic control behavior of nonlinear dynamics of encapsulated microbubble. It should be noted that controlling the chaotic oscillations of microbubbles in ultrasonic fields is studied without requiring any knowledge about the state of system. This method is simple and easy to implement experimentally. Also, it can be easily applied to a bubble cluster in an ultrasonic field [73].

\section{Appendix A. Stability analysis}

The first-order differential equations for the specific example (Eq. 1) are of the following form:

$$
\left\{\begin{array}{l}
\dot{x}=\frac{y}{x_{0}} \\
\dot{y}=\left[\frac{3}{2}\left[(3 \beta+1) \frac{y}{3 c}-1\right] y^{2}+\frac{12 \mu_{s h} \epsilon}{\rho c\left(x_{0} x-\epsilon\right) x_{0} x}\left[\left(\beta+\frac{x_{0} x}{x_{0} x-\epsilon}\right) \frac{y}{c}-1\right]\right. \\
y-\frac{1}{\rho}\left(\frac{2 \sigma}{x_{0}}+P_{0}\right)\left[\left(1+(1-\beta) \frac{y}{c}\right)\right]+\frac{1}{\rho}\left(\frac{2 \sigma+\chi}{x_{0}}+P_{0}\right)\left[\left(1+(1-\beta-3 \Gamma) \frac{y}{c}\right)\right] \\
\left(\frac{1}{x}\right)^{3 \Gamma}+\frac{4 \mu}{x_{0} x \rho}\left(\frac{\beta y}{c}-1\right) y+\frac{2 \chi}{x_{0} x \rho}\left[(2+\beta) \frac{y}{c}-1\right] \\
\left.\left(\frac{1}{x}\right)^{2}-\frac{1}{\rho}\left[1+(1-\beta) \frac{y}{c}\right] P_{a} \sin (2 \pi \theta)-\frac{2 \pi f x}{\rho c} P_{a} \cos (2 \pi \theta)\right] \\
/\left[\left(1-(\beta+1) \frac{y}{c}\right) x_{0} x+\frac{4 \mu}{\rho c}+\frac{12 \mu_{s h} \epsilon}{\rho c\left(x_{0} x-\epsilon\right)}\right] \\
\dot{\theta}=f .
\end{array}\right.
$$

where $\theta$ is the cyclic variable. Hence the first-order differential equations for the specific example (Eq. 1) with dynamical parameter are of the following form: 


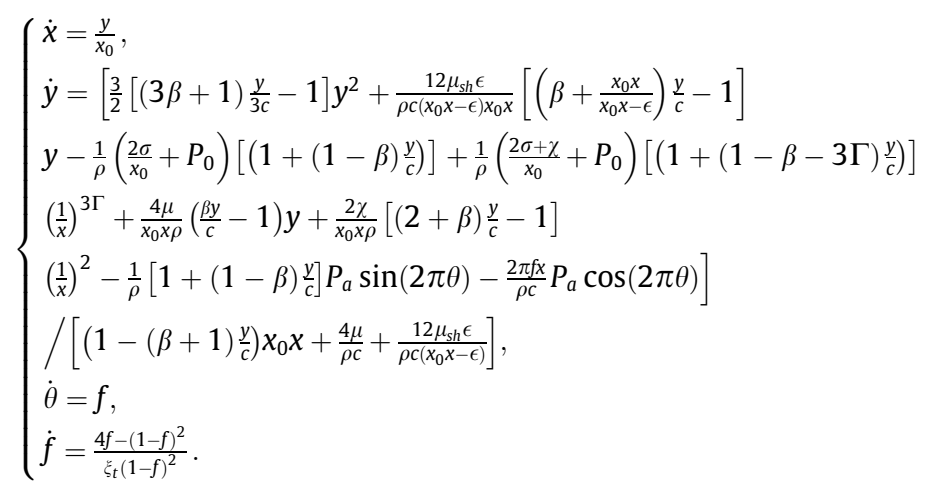

\section{References}

[1] M.J. Borrelli, W.D. O'Brien Jr, et al, Production of uniformly sized serum albumin and dextrose microbubbles, J. Ultrasound Med. 19 (2012) $198-208$.

[2] S.R. Sirsi, M.A. Borden, Microbubble compositions, properties and biomedical applications, Bubble Sci. Eng. Technol. 1 (2009) 3-17.

[3] P. Decuzzi, B. Godin, T. Tanaka, S.Y. Lee, C. Chiappini, X. Liu, M. Ferrari, Size and shape effects in the biodistribution of intravascularly injected particles, J. Controlled Release 141 (2010) 320-327.

[4] M. Postema, G. Schmitz, Ultrasonic bubbles in medicine: influence of the shell, Adv. Drug Delivery Rev. 14 (2007) 438-444.

[5] K. Hynynen, Ultrasound for drug and gene delivery to the brain, Adv. Drug Delivery Rev. 60 (2008) 1209-1217.

[6] J.H. Hwang, S. Vaezy, R.W. Martin, et al, High-intensity focused us: a potential new treatment for Gi bleeding, Gastrointest. Endosc. 58 (2003) 111-115.

[7] S.R. Sirsia, S.L. Hernandezb, et al, Polyplex-microbubble hybrids for ultrasound-guided plasmid dna delivery to solid tumors, J. Controlled Release 157 (2011) 224-234.

[8] J. Pepe, M. Rincon, J. Wu, Experimental comparison of sonoporation and electroporation in cell transfection applications, Acoust. Res. Lett. 5 (2004) 6267.

[9] C.C. Coussios, C.H. Farny, G. Ter Haar, R.A. Roy, Role of acoustic cavitation in the delivery and monitoring of cancer treatment by high-intensity focused ultrasound (hifu), Int. J. Hypertherm. 23 (2007) 105-120.

[10] K. Iwanaga, K. Tominaga, K. Yamamoto, et al, Local delivery system of cytotoxic agents to tumors by focused sonoporation, Cancer Gene Ther. 14 (2007) $354-363$.

[11] W. Hundt, E. Yuh, M.D. Bednarski, S. Guccione, Gene expression profiles, histologic analysis, and imaging of squamous cell carcinoma model treated with focused ultrasound beams, AJR, Am. J. Roentgenol. 189 (2007) 726-736.

[12] C.A. Molina, M. Ribo, M. Rubiera, et al, Microbubble administration accelerates clot lysis during continuous 2-mhz ultrasound monitoring in stroke patients treated with intravenous tissue plasminogen activator, Stroke 37 (2006) 425-429.

[13] P. Marmottant, S. Hilgenfeldt, Controlled vesicle deformation and lysis by single oscillating bubbles, Nature 423 (2003) 153-156.

[14] T.M. Poster, D.A.B. Smith, C.K. Holland, Acoustic techniques for assessing the optison destruction threshold, J. Ultrasound Med. 25 (2006) $1519-1529$.

[15] F. Wu, Z.B. Wang, W.Z. Chen, et al, Extracorporeal high intensity focused ultrasound ablation in the treatment of 1038 patients with solid carcinomas in china: an overview, Ultrason. Sonochem. 11 (2004) 149-154.

[16] D. Razansky, P.D. Einziger, et al, Enhanced heat deposition using ultrasound contrast agent-modeling and experimental observations, IEEE Trans. UFFC 53 (2006) 137-147.

[17] S. Umemura, K. Kawabata, K. Sasaki, In vivo acceleration of ultrasonic tissue heating by microbubble agent, IEEE Trans. Ultrason. Ferroelectr. Freq. Control 52 (2005) 1690-1698.

[18] Y. Kaneko et al, Use of a microbubble agent to increase the effects of high intensity focused ultrasound on liver tissue, Eur. Radiol. 15 (2005) 14151420.

[19] W. Luo, X. Zhou, X. Ren, M. Zheng, J. Zhang, G. He, Enhancing effect of sonovue, a microbubble sonographic contrast agent, on high-intensity focused ultrasound ablation in rabbit livers in vivo, J. Ultrasound Med. 26 (2007) 469-476.

[20] D.J. Chunga, S.H. Cho, J.M. Leea, S-T. Hahna, Effect of microbubble contrast agent during high intensity focused ultrasound ablation on rabbit liver in vivo, Eur. J. Radiol. 81 (2012) e519-e523.

[21] K. Kajiyama, K. Yoshinaka, S. Takagi, Y. Matsumoto, Micro-bubble enhanced hifu, Phys. Proc. 3 (2010) 305-314.

[22] T.G. Leighton, The Acoustic Bubble, Academic, London, 1994.

[23] A. Prosperetti, A. Lezzi, Bubble Dynamics in a Compressible Liquid. Part 1: First-Order Theory, vol. 168, Academic Press Limited, London, 1986. 457478.

[24] N. de Jong, R. Cornet, C.T. Lancee, Higher harmonics of vibrating gas-filled microspheres. Part one: Simulations, Ultrasonics 32 (1994) 447-453.

[25] C.C. Church, The effects of an elastic solid surface layer on the radial pulsations of gas bubbles, J. Acoust. Soc. Am. 97 (1995) 1510-1521.

[26] K.E. Morgan, J.S. Allen, P.A. Dayton, J.E. Chomas, A.L. Klibanov, K.W. Ferrara, Experimental and theoretical evaluation of microbubble behaviour: effect of transmitted phase and bubble size, IEEE Trans. Ultrason. Ferroelectr. Freq. Control 47 (2000) 1494-1509.

[27] K. Vokurka, Comparison of Rayleigh's, Herring's and Gilmore's models of gas bubbles, Acustica 59 (1986) 214-219.

[28] R.E. Glazman, Effects of absorbed films on gas bubble radial oscillations, J. Acoust. Soc. Am. 74 (1983) 980-986.

[29] C.A. MacDonald, V. Sboros, J. Gomatam, S.D. Pye, C.M. Moran, W.N. McDicken, A numerical investigation of the resonance of gas-filled microbubbles: resonance dependence on acoustic pressure amplitude, Ultrasonics 43 (2004) 113-122.

[30] M. Fischer, I. Zinovik, D. Poulikakos, Diffusion and reaction controlled dissolution of oxygen microbubbles in blood, Int. J. Heat Mass Transfer 52 (2009) 5013-5019.

[31] A.A. Aganin, Dynamics of a small bubble in a compressible fluid, Int. J. Numer. Methods Fluids 33 (2000) 157-174.

[32] P.J. Harris, A numerical method for modelling the motion of a spherical bubble, Int. J. Numer. Methods Fluids 22 (1996) 1125-1134.

[33] S. Behnia, A. Akhshani, Dynamical control of chaos by Slave-Master feedback, Chaos Solitons Fract. 42 (2009) 2105-2114.

[34] M.A. Jafarizadeh, S. Behnia, S. Khorram, H. Nagshara, Hierarchy of chaotic maps with an invariant measure, J. Stat. Phys. 104 (2001) $1013-1028$.

[35] C.A. Macdonald, J. Gomatam, Chaotic dynamics of microbubbles in ultrasonic fields, Proc. IMech. E 220 (2006) 333-343.

[36] K.E. Morgan, J.S. Allen, P.A. Dayton, J.E. Chomas, A.L. Klibanov, K.W. Ferrara, Experimental behaviour: effect of transmitted phase and bubble size, IEEE Trans. Ultrason. Ferroelectr. Freq. Control 47 (2000) 1494-1509.

[37] Y. Sun, D.E. Kruse, P.A. Dayton, K.W. Ferrara, Highfrequency dynamics of ultrasound contrast agents, IEEE Trans. Ultrason. Ferroelect. Freq. Contr 52 (2005) 1981-1991.

[38] E.C. Unger, T.O. Matsunaga, T. McCreery, P. Schumann, R. Sweitzer, R. Quigley, Therapeutic applications of microbubbles, Eur. J. Radiol. 42 (2002) $160-$ 168. 
[39] K. Vokurka, Comparison of Rayleigh's, Herring's and Gilmore’s models of gas bubbles, Acustica 59 (1986) $214-219$.

[40] K.J.Y. Chong, C.Y. Quek, F. Dzaharudin, A. Ooi, R. Manasseh, The effcts of coupling and bubble size on the dynamical-systems behaviour of a small cluster of microbubbles, J. Sound Vibr. 329 (2010) 687-699.

[41] E. Stride, M-X. Tang, R.J. Eckersley, Physical phenomena affecting quantitative imaging of ultrasound contrast agents, Appl. Acoust. 70 (2009) 13521362.

[42] J. Jiménez-Fernndez, Nonlinear response to ultrasound of encapsulated microbubbles, Ultrasonics 52 (2012) $784-793$.

[43] S. Behnia, A. Jafari, W. Soltanpoor, O. Jahanbakhsh, Nonlinear transitions of a spherical cavitation bubble, Chaos Solitons Fract. 41 (2009) $818-828$.

[44] I.S. Akhatov, S.I. Konovalova, Regular and chaotic dynamics of a spherical bubble, J. Appl. Math. Mech. 69 (2005) $575-585$.

[45] W. Lauterborn, L. Cramer, Subharmonic route to chaos observed in acoustics, Phys. Rev. Lett. 47 (1981) $1445-1448$.

[46] W. Lauterborn, A. Koch, Holographic observation of period-doubled and chaotic bubble oscillations in acoustic, Phys. Rev. A 35 (1987) $1974-1976$.

[47] R.G. Holt, D.F. Gaitan, A.A. Atchley, J. Holzfuss, Chaotic sonoluminescence, Phys. Rev. Lett. 72 (1994) $1376-1379$.

[48] S. Behnia, A. Jafari Sojahrood, W. Soltanpoor, O. Jahanbakhsh, Suppressing chaotic oscillations of a spherical cavitation bubble through applying a periodic perturbation, Ultrason. Sonochem. 16 (2009) 502-511.

[49] E. Ott, C. Grebogi, J.A. Yorke, Controlling chaos, Phys. Rev. Lett. 64 (1990) 1196-1199.

[50] E.G. Chen, Controlling Chaos and Bifurcations in Engineering Systems, CRC Press, 1999.

[51] M. Ding, W. Yang, V. In, W.L. Ditto, M.L. Spano, B. Gluckman, Controlling chaos in high dimensions: theory and experiment, Phys. Rev. E 53 (1996) 4334-4344.

[52] R. Li, W. Xu, S. Li, Chaos controlling of extended nonlinear Linard system based on the Melnikov theory, Appl. Math. Comput. 178 (2006) $405-414$.

[53] Y.-C. Hung, T.-L. Liao, J.-J. Yan, Adaptive variable structure control for chaos suppression of unified chaotic systems, Appl. Math. Comput. 209 (2009) 391-398.

[54] K. Pyragas, Continuous control of chaos by self-controlling feedback, Phys. Lett. A 170 (1992) $421-428$.

[55] R. Meucci, D. Cinotti, E. Allaria, L. Billings, I. Triandaf, D. Morgan, I.B. Schwartz, Global manifold control in a driven laser: sustaining chaos and regular dynamics, Physica D 189 (2004) 70-80.

[56] U. Parlitz, V. Englisch, C. Scheffczyk, W. Lauterborn, Bifurcation structure of bubble oscillators, J. Acoust. Soc. Am. 88 (1990) $1061-1077$.

[57] J.R. Dorfman, An Introduction to Chaos in Nonequilibrium Statistical Mechanics, Cambridge University Press, New York, 1999.

[58] E. Ott, Chaos in Dynamical System, University Press, New York, 2002.

[59] A. Wolf, J.B. Swift, H.L. Swinney, A. Vastano, Determining lyapunov exponents from a time series, Physica D 16D (1985) $285-317$.

[60] G. Simon, P. Cvitanovic, M.T. Levinsen, I. Csabai, A. Horath, Periodic orbit theory applied to a chaotically oscillating gas bubble in water, Nonlinearity 15 (2002) 25-43.

[61] W. Lauterborn, U. Parlitz, Methods of chaos physics and their application to acoustics p bifurcation structure of bubble oscillators, J. Acoust. Soc. Am. 84 (1988) 1975-1993.

[62] Y. Kaneko, T. Maruyama, et al, Use of a microbubble agent to increase the effects of high intensity focused ultrasound on liver tissue, Eur. Radiol. 15 (2005) 14151420.

[63] Z. Kyriakou et al, Hifu-induced cavitation and heating in ex vivo porcine subcutaneous fat, Ultrasound Med. .Biol. 37 (2011) $568-579$.

[64] S. Peng, Y. Xiong,ith high intensity focused ultrasound: A retrospective study, European Journal of Radiology 2012; In press.

[65] T. Yu, S. Xiong, T.J. Mason, Z. Wang, The use of a microbubble agent to enhance rabbit liver destruction using high intensity focused ultrasound, Ultrason. Sonochem. 13 (2006) 143-149.

[66] T.M. Poster, D.A.B. Smith, et al, Acoustic techniques for assessing the optison destruction threshold, J. Ultrasound Med. 25 (2006) 1519-1529.

[67] F. Wu, Z.B. Wang, W.Z. Chen, et al, Extracorporeal high intensity focused ultrasound ablation in the treatment of 1038 patients with solid carcinomas in china: an overview, Ultrason. Sonochem. 11 (2004) 149-154.

[68] D. Razansky, P.D. Einziger, et al, Enhanced heat deposition using ultrasound contrast agent-modeling and experimental observations, IEEE Trans. UFFC 53 (2006) 137-147.

[69] S. Umemura, K. Kawabata, K. Sasaki, Enhancement of sonodynamic tissue damage production by second-harmonic superimposition: theoretical analysis of its mechanism, IEEE. Trans. Ultrason. Ferroelect. Freq. Contr. 43 (1996) 1054-1062.

[70] N.J. McDannold, N. Vykhodtseva, K. Hynynen, Microbubble contrast agent with focused ultrasound to create brain lesions at low power levels: Mr imaging and histologic study in rabbits, Radiology 241 (2006) 95-106.

[71] Y.L. Wen, M. Kudo, R.Q. Zheng, et al, Radiofrequency ablation of hepatocellular carcinoma: therapeutic response using contrast-enhanced coded phaseinversion harmonic sonography, Am. J. Roentgenol. 181 (2003) 57-63.

[72] J.E. Kennedy, G.R. ter Haar, F. Wu, et al, Contrast-enhanced ultrasound assessment of tissue response to high-intensity focused ultrasound, Ultrasound Med. Biol. 30 (2004) 851-854.

[73] Y. Matsumoto, S. Yoshizawa, Behaviour of a bubble cluster in an ultrasound field, Int. J. Numer. Methods Fluids 47 (2005) $591-601$. 